\title{
Just a Bit of Physics Can Tell So Much: A Unique Story of the Start of the Earth-Moon System
}

\author{
Fred J. Cadieu \\ Department of Physics, Queens College of CUNY, New York, NY, USA \\ Email: fred.cadieu@qc.cuny.edu
}

How to cite this paper: Cadieu, F.J. (2020) Just a Bit of Physics Can Tell So Much: A Unique Story of the Start of the EarthMoon System. World Journal of Condensed Matter Physics, 10, 88-103. https://doi.org/10.4236/wjcmp.2020.102006

Received: March 6, 2020

Accepted: May 17, 2020

Published: May 20, 2020

Copyright $\odot 2020$ by author(s) and Scientific Research Publishing Inc. This work is licensed under the Creative Commons Attribution International License (CC BY 4.0).

http://creativecommons.org/licenses/by/4.0/

(c) (i) Open Access

\begin{abstract}
The start of the Earth-Moon system has been studied to show that this was an exceptionally violent event. One result was that Earth became the terrestrial planet with the highest average density. Another result was that Earth acquired enough mass and radioactive elements that it is expected to maintain a molten core region and magnetic field for the expected life of the Earth. Earth alone of the terrestrial planets was then able to develop plate tectonics as a long term energy release mechanism. The dipole magnetic field of the Sun reverses periodically, currently at the rate of about every 11 years, so that there was a magnetic braking action acting on the core of Venus that accounts for the slow rotation of that planet. A key result is that the impact event that resulted in the Earth-Moon system led to long term stability on Earth that allowed the eventual development of complex life forms on the Earth.
\end{abstract}

\section{Keywords}

Earth, Moon, Magnetic Fields Sun, Venus, Plate Tectonics

\section{Introduction}

The currently most widely accepted view of the start of the Earth-Moon system is that there was an oblique collision between a pre-Earth and an approximately Mars-sized planet that was forming near the orbit of the pre-Earth [1]. This collision occurred at approximately 20 - 100 million years after the initial formation of the solar system. The time period of this collision has been determined by dating the Earth and the moon. The consequences of this formation of the Moon as a relatively large satellite of the Earth have only recently become clearer. In this paper, we want to use a bit of physics to extrapolate back in time to more fully explore the development of the terrestrial planets in our solar system. The 
purpose of this paper is to illuminate how the formation of the Earth-Moon system played a crucial role in Earth exhibiting a magnetic field, plate tectonics, and long term evolution to allow the evolution of complex life forms.

\section{Results}

The first step will be to explore why the same face of the Moon always faces toward the Earth. The rotation of the Earth and Moon can be traced back to earlier times by conserving angular momentum as the Moon gradually moved away from the Earth. To see why a satellite would circle the Earth with the same side facing toward the Earth, objects that circle the Earth can be studied with the idea that a reason can then be generalized to the Moon and other satellites. As a first step, we can consider what orientation the space shuttle would assume as it circled the Earth as a near Earth satellite with a period of about 90 minutes as determined by Newton's Laws. Would it circle the Earth with the nose pointing down toward the Earth, pointed up away, or fly level like an airplane. This is illustrated in Figure 1. A quick examination shows that this is not a fundamental question since the shuttle, although an elongated structure, has a large cargo bay so that the location of the center of mass can be changed depending on the mass distribution of different cargos, and besides the space shuttle had a set of rockets mounted on the nose that could be used to change and reorient the spatial orientation as the space shuttle orbited the Earth. It then becomes useful to consider the orbit orientation of an artificial body that can be precisely defined. For this purpose, an artificial structure has been defined called the FedEx arrow that consists of a rectangle-shaped 4 units by 2 units as the tail of an equilateral triangle of side 4 units. For simplicity, it is assumed that the rectangle and triangle are both 1 unit thick. Such a structure is illustrated in Figure 2. The rectangle and the triangle have the same surface density and are welded together so that the center of mass of both parts and of the composite object can be easily determined. This is an elongated structure as with the space shuttle, but now the orbit orientation can be determined by finding the spatial orientation that yields the lowest gravitational potential energy. For the structure shown in Figure 2, the

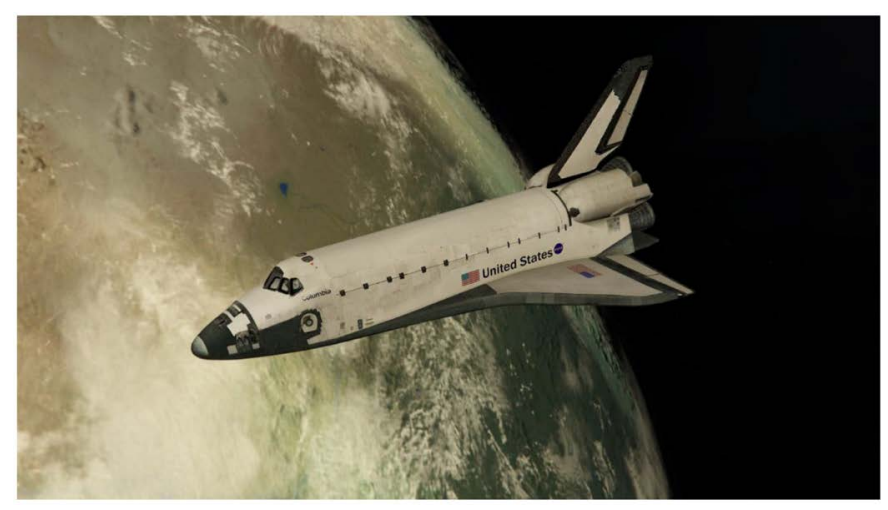

Figure 1. The space shuttle used to circle the Earth in a near earth orbit. The spatial orientation was controllable by a set of rockets (NASA image). 


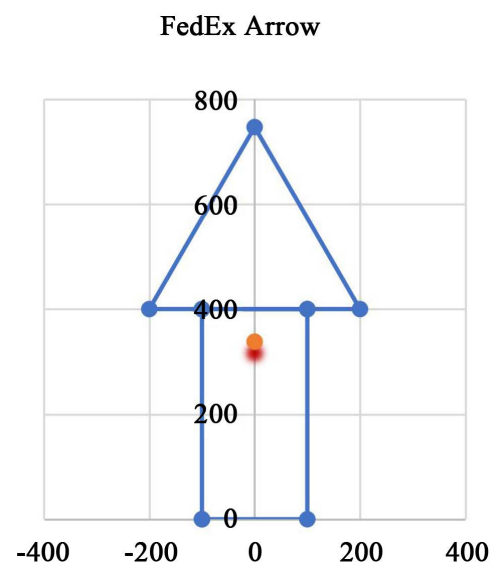

Figure 2. This object called FedEx Arrow is composed of a rectangle and an equilateral triangle so that Newton's law of gravity can be used to minimize the gravitational potential energy to determine the preferred spatial orientation.

center of mass of the rectangle is at $(0,200)$, and center of mass of the triangle at $(0,515)$, and the center of mass of the composite object is at $(0,346)$. The lowest gravitational potential energy occurs when the arrow is pointing toward the Earth as the FedEx arrow orbits the Earth. This is shown in Appendix. The distance from the center of mass of the composite object to the center of mass of the Earth is the relevant distance for Newton's laws. Here it has been assumed that the mass of the Earth is very much larger than the mass of the FedEx arrow so that the motion has been taken to be about the center of the Earth.

To continue with a discussion of how the Earth-Moon system also relates to the development of the neighboring terrestrial planets some basic facts about the solar system should be recalled.

\section{Background of the Solar System}

- The mass of the Sun is about a million times the mass of the Earth.

- The mass of Jupiter alone is 320 times the mass of the Earth.

- $99.9 \%$ of the solar system mass resides in the Sun.

- The Sun acts on the Planets through.

1) The Force of Gravity: $F=G \frac{M_{S}}{R_{c m}^{2}} M_{P}$ where $G$ is the gravitational constant, $M_{S}$ is the mass of the Sun, $M_{P}$ is the mass of a particular planet, and $R_{c m}$ is the distance from the center of mass of the Sun to the center of mass of the planet.

2) Light-Luminosity $L \approx L_{S} \cdot\left(0.7+\frac{0.3 \cdot t}{4.6 B y r}\right)$ so that the Sun is gradually getting brighter [2].

3) Magnetic Field-Early on in the formation of the solar system the Sun's rotating magnetic field provided a mechanism for transferring angular momentum away from the Sun so that everything did not just collapse into the Sun.

4) The Solar Wind-There is a stream of charged ions and electrons streaming away from the Sun.

- All of these terrestrial planets and the moon were large enough to have as- 
sumed a spherical shape because of their self-gravitational attraction.

- Compaction, heating, and segregation by gravity occurred.

- Core size, core circulation, core conductivity, and longevity of any state depends on the initial size, radioactivity, thermal and electrical conductivity.

- The Earth has a molten core region and a magnetic field created due to rotation of an outer liquid core-Basic question: Did Mercury, Venus, or Mars ever exhibit a similar magnetic field to that of the Earth?

Figure 3 diagrammatically depicts the oblique collision that resulted in the Earth-Moon system. The extent of mass transfer and angular momentum of the resulting system depends on the details of the collision. But there will be a debris field about the new Earth while the debris is within the Roche limit. The Roche limit for the Earth is about 3 Earth radii [3]. As the debris field moved out from the Earth it coalesced into the present Moon. The time period of the interaction is thought to take only several weeks. As the moon forms, it is expected to be an elongated mass distribution due to the interaction shown in Figure 4. Just as with the FedEx arrow as shown earlier the Moon then continued to orbit the Earth with the same side facing toward the Earth. Support for this Moon orientation can be found in the planet information site NinePlanets.org where it states: "Curiously, the Moon's center of mass is offset from its geometric center by about $2 \mathrm{~km}$ in the direction toward the Earth". That the interaction between the pre-Earth and impactor was particularly violent can be noted from the expected mass ratio and pre-Earth being approximately 8 to 1 for a Mars size impactor versus the present mass ratio of the Earth to the moon being 81.3 [2]. Over time the side facing the Earth was shielded by the Earth so that the far side is expected to have accumulated far more crater impacts. This initial elongation causing event, and the resulting same side of the Moon then continually facing the Earth resulted in the different evolution of the two sides of the Moon.

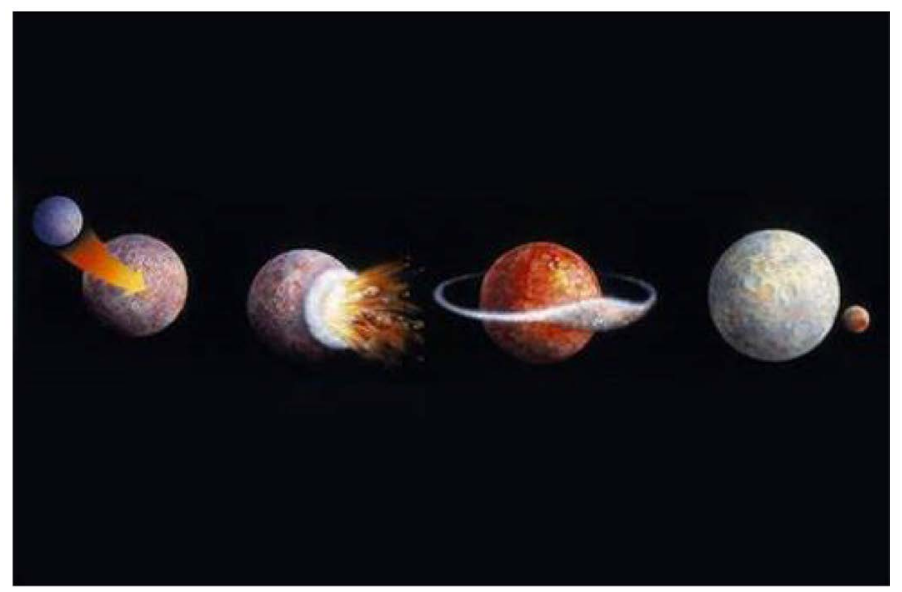

Figure 3. The pre-earth and impactor violently collided and much of the impactor mass was transferred to the earth. The debris field collected into the Moon only as the debris field started to move beyond the Roche limit. The expected mass ratio for a Mars sized impactor would be $8: 1$. That the Earth to Moon ratio is actually 81.3 to one is indicative of the violence of the collision (museum.victoria.com.au image). 


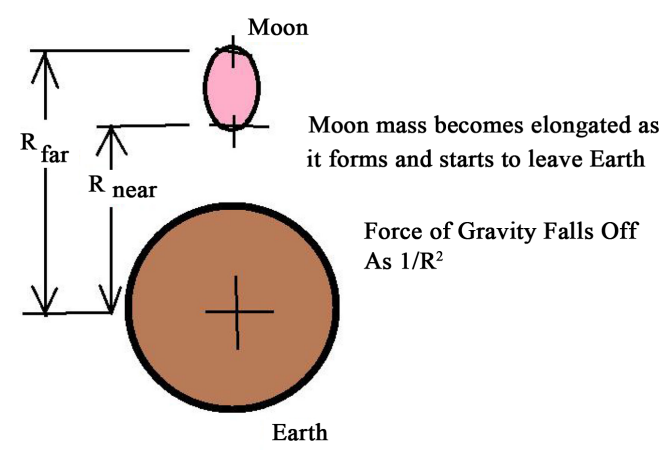

Figure 4. The moon became elongated as the moon started to separate from the Earth.

Figure 5 shows the familiar face of the Moon that always faces the Earth. The angular momentum of the Earth-Moon orbital rotation consists of three terms including the rotation of the Earth on its rotation axis, the rotation of the Moon on its rotation axis, and the rotation of the Moon about the Earth. Since the Earth to Moon mass ratio is 81.3:1 the approximation is made that the center of mass of the Earth-Moon is nearly at the center of the Earth to simplify the calculation of how the Earth-Moon system evolved over the time since formation. The Earth-Moon as a system orbits the Sun, but that has little effect on the calculations presented here. Since the same side of the Moon always faces the Earth, the period of the Moon about the Earth equals the period of the Moon about its rotation axis. Application of Kepler's third law to the Earth-Moon system allows the motion to be extrapolated either back in time, or to be projected into the future. The present Earth rotation period of 24 hours at an Earth Moon distance of $3.84 \times 10^{5} \mathrm{~km}$ can then be used as a known point to allow extrapolations backward as well as forward in time (Table 1 ).

Figure 6 shows the Earth day length versus the Earth-Moon distance that results from such a conservation of angular momentum calculation. For simplicity, the orbit of the Moon about the Earth has been approximated as circular. Early on, the length of a day was only about 5 hours. Early tides would have been horrific. Recently, an article suggests that continents and the start of land extending above the Earth covering ocean until after about 1.4 billion years had elapsed [4]. Tidal turbulence and tidal friction should have made the surface of the Earth relatively warm. Not until the Moon had moved out to about $2 \times 10^{8} \mathrm{~m}$ from the Earth would the time between high tides lengthen to about 5 hours. At the present Earth-Moon distance of $3.84 \times 10^{5} \mathrm{~km}$ the time between high tides has lengthened to about 6.4 hours. That this time is greater than 6 hours means that the time of high tide keeps changing from day to day. Figure 7 shows that the period of the Moon about the Earth did not lengthen to about 15 days until after the first 2.5 billion years.

The annotations in Figure 8 illustrate just how long a billion years is, it may be noted that today the length of a day is 24 hours, but at the time of earliest mankind, about 200 thousand years ago, the day was still about 23.92 hours long. But at the time of the dinosaurs about 200 million years ago the day was 


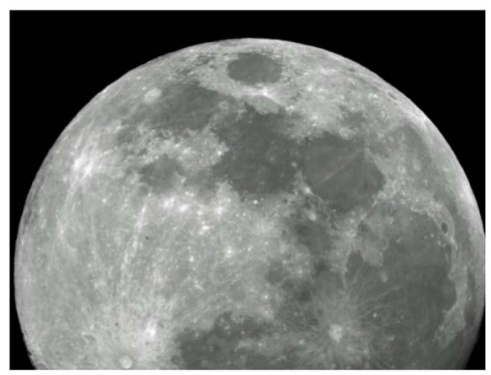

Figure 5. The familiar face of the Moon that faces the Earth is shown. Photograph taken by author.

Earth to Moon Distance versus Length of Day on Earth

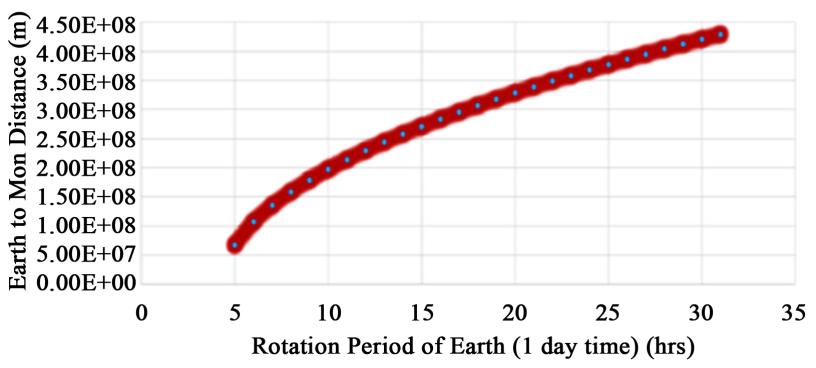

Figure 6. This figure shows the length of an earth day versus the earth to moon distance.

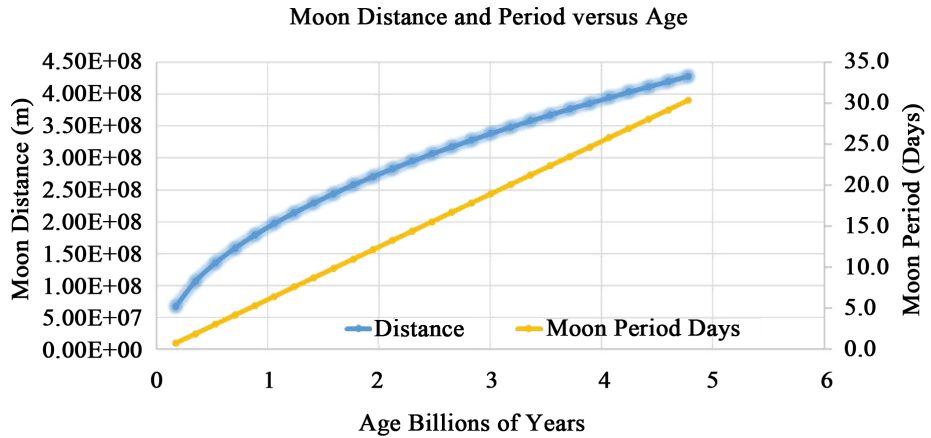

Figure 7. The earth-moon distance (blue trace) and Moon period (yellow trace) versus age is shown.

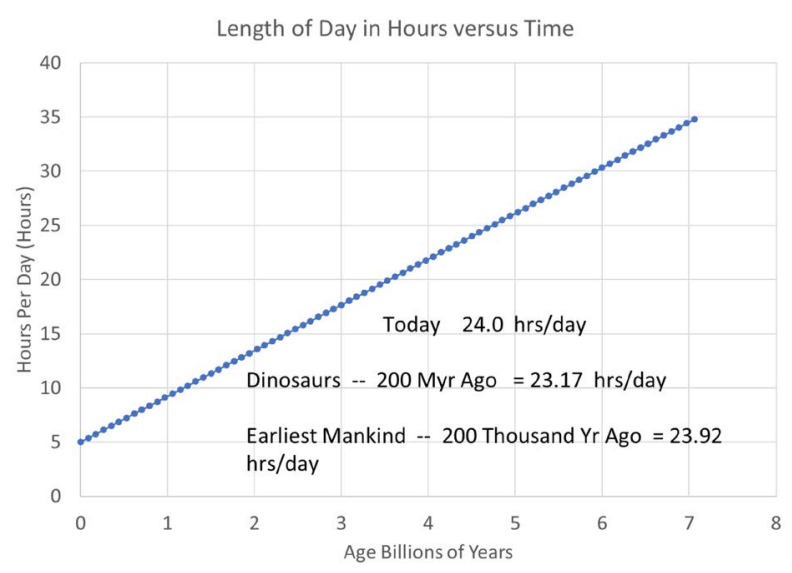

Figure 8. The earth day in hours versus earth age is shown. 
Table 1. NASA planet details [5].

\begin{tabular}{|c|c|c|c|c|c|c|c|}
\hline Planet & $\begin{array}{c}\text { Mass } \\
(\text { Earth = 1) }\end{array}$ & $\begin{array}{c}\text { Semimajor } \\
\text { Orbit } \\
\text { Radius } \\
\text { (AU) }\end{array}$ & Eccentricity & $\begin{array}{l}\text { Orbit } \\
\text { Period } \\
\text { (Solar } \\
\text { Days) }\end{array}$ & $\begin{array}{c}\text { Rotation } \\
\text { Period } \\
\text { (Solar } \\
\text { Days) }\end{array}$ & $\begin{array}{c}\text { Orbit } \\
\text { Tilt } \\
\text { Angle }\left(^{\circ}\right)\end{array}$ & $\begin{array}{c}\text { Average } \\
\text { Density } \\
(\text { Earth = 1) }\end{array}$ \\
\hline "Sun" & 332,000 & & & & & & 0.254 \\
\hline Mercury & 0.055 & 0.39 & 0.206 & 88.0 & $\begin{array}{c}58.6(3: 2 \\
\text { orbital to } \\
\text { rotation } \\
\text { locking) }\end{array}$ & 7.0 & 0.98 \\
\hline Venus & 0.82 & 0.72 & 0.007 & 224.7 & -243.0 & 3.39 & 0.95 \\
\hline Earth & 1 & 1.00 & 0.017 & 365.26 & 0.9973 & 23.45 & 1.00 \\
\hline Mars & 0.11 & 1.52 & 0.093 & 686.9 & 1.026 & 25 & 0.71 \\
\hline Jupiter & 317.8 & 5.20 & 0.048 & $\begin{array}{c}11.86 \\
\text { tropical } \\
\text { years }\end{array}$ & 0.41 & 1.31 & 0.241 \\
\hline $\begin{array}{l}\text { "Moon orbit } \\
\text { about Earth" }\end{array}$ & 0.012 & $2.567 \times 10^{-3}$ & 0.055 & 27.32 & 27.32 & $\begin{array}{l}5.2 \text { relative } \\
\text { to Earth } \\
\text { orbit }\end{array}$ & 0.61 \\
\hline
\end{tabular}

only 23.17 hours long. Over the time of humanoids, 250,000 years, the Earth-Moon distance has hardly changed!

\section{Discussions}

To see how the Earth-Moon system relates to the development of the other terrestrial planets in the solar system it helps to consider the development of the inner solar system since its initial formation. It is possible to look far back to this initial formation because our solar system exhibits what may be termed a friendly Jupiter. This friendly Jupiter concept means that a large gas giant planet exhibits a nearly circular orbit that allowed the inner solar system to develop with certain regularities that persisted from the early solar system formation (Table 1). One such regularity, is that there is an expectation that the average densities of the terrestrial planets can be expected to decrease as the temperature decreases as the formation zone of a planet is positioned at greater distances away from the Sun. This expectation is illustrated in Figure 9 which shows the average terrestrial planet density as a function of the distance away from the Sun. The fact that our solar system has a friendly Jupiter has allowed this density relationship to have persisted over the last 4.5 billion years. It should be noticed that Mercury and Venus do not have any satellite moons, and that Mars only has a few larger rocks as satellites. Thus the three terrestrial planets Mercury, Venus, and Mars allow the plotting of the line shown in Figure 9 (Table 1). The expected density of the pre-Earth and pre-Moon can then be determined as the density of a terrestrial planet for a distance of about 1 astronomical unit, $1 \mathrm{AU}$, from the Sun. For the Earth to result from an oblique collision of the pre-Earth as being an approximately Mars sized object with an approximate size of $1 / 2$ the 


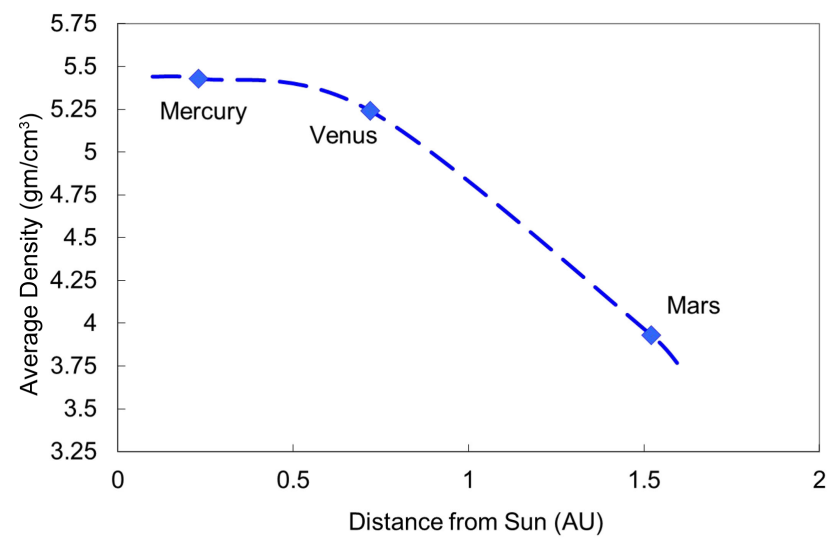

Figure 9. The average density of the terrestrial planets Mercury, Venus and Mars versus distance from the Sun is shown.

pre-Earth diameter gives an expected mass ratio of 8 to 1 since the volume is proportional to the radius cubed. The fact that the final Earth to Moon mass ratio is 81 to 1 instead of 8 to 1 indicates the interaction was very violent and that a large fraction of the mass and mass distribution of the pre-Moon was transferred to the resultant Earth. The resultant average densities including the Earth and moon are illustrated in Figure 10 (Table 1). An important result of this collision was that the Earth, rather unexpectedly, became the terrestrial planet with the highest average density!

The terrestrial planets formed by converting gravitational potential energy into differentiated bodies with enough heat involved to cause hot interior cores. During the compaction process, there is the expectation that a large fraction of water would be boiled off. Comets carrying water back to the terrestrial planets provides a mechanism that yields surface oceans. For a condition of rapid bombardment as expected during the early inner solar system formation there is the expectation that the number of impacts was proportional to the planet cross section which is proportional to the radius squared. And since the surface area for water distribution is also proportional to the radius squared, the ocean depths on the different planets are expected to be the same. The subsequent changes in the ocean levels depend largely on the planet size, surface temperatures, and proximity to the Sun as an energy source. A key parameter controlling these interactions is the vapor pressure of water as a function of temperature as shown in Figure 11. The vapor pressure of water is highly nonlinear so that small differences in temperature can cause large differences in changes of water retention and evolution. For an approximate early temperature on Earth of $25^{\circ} \mathrm{C}$, a corresponding temperature on Venus would be about $50^{\circ} \mathrm{C}$ greater than this because of the closer proximity to the Sun even neglecting any atmospheric effects. Consequently, at the higher surface temperature of Venus, the vapor pressure of water would be about an order of magnitude greater than on Earth so a much greater rate of water evaporation into the upper atmosphere was expected on Venus. 


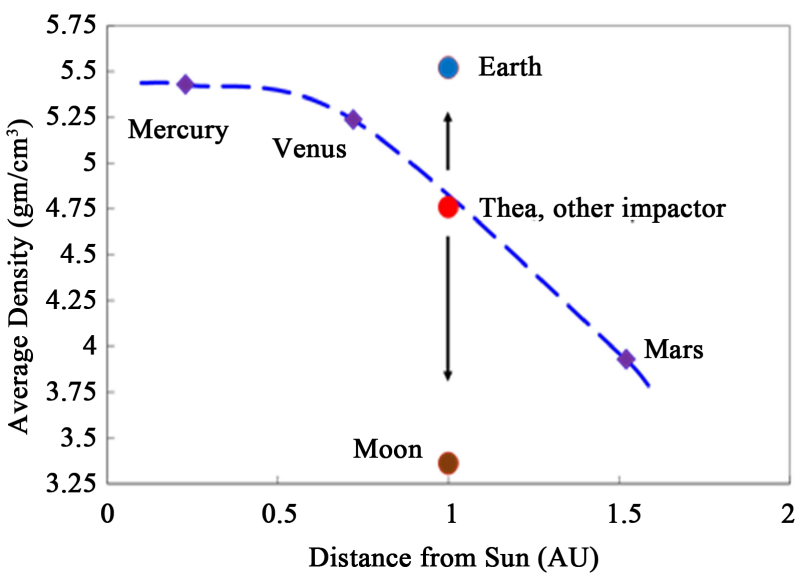

Figure 10. Projected and measured average densities of earth and the moon are plotted. Earth becomes densest of the terrestrial planets!

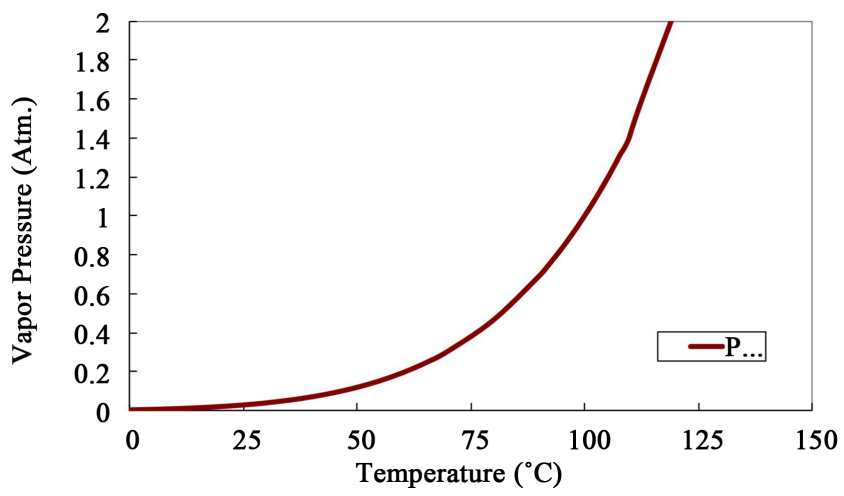

Figure 11. The vapor pressure of water versus temperature is shown.

For Venus, being closer to the Sun, the surface temperature is expected to be about $50^{\circ} \mathrm{C}$ warmer than Earth. A larger fraction of the surface water on Venus can then be expected to be vaporized to the upper atmosphere where it can be disassociated by ultraviolet light from the Sun. The hydrogen can then escape to space since there were few other types of molecules present to bond with the hydrogen. Over time hydrogen and thus water was lost to space. In addition, since Venus was not exhibiting a magnetic field the solar wind was also acting to strip away the upper atmosphere. For Earth, being at a lower temperature, a smaller fraction of water reached the upper atmosphere and the Earth's magnetosphere protected it from the solar wind. It was crucial that the Earth's surface stayed relatively cool to limit water evaporation until Earth's atmosphere could include other molecules such as oxygen and ozone that could interact with disassociated hydrogen to prevent it from escaping to space. Earth did not develop an oxygen rich atmosphere until after about $2.2 \mathrm{BYr}$ [6]. It should be noted that this represents a time period of about $48 \%$ the age of the present Earth. Long periods of sequential evolutionary development had to occur to allow this oxygen rich event to have occurred. This indicates that Earth experienced long term stability that only occurs with plate tectonics to allow gradual heat releases. 
This unexpectedly high density of the Earth as shown in Figure 10 has a large number of direct developmental consequences for the Earth that also distinguish it from Mercury, Venus and Mars. In progressing out from the Sun, Mercury and Venus could be expected to develop a differentiated structure with a metallic electrically conducting core. Mercury has an equatorial radius of 0.38 that of Earth so that the interior solidified early in its history and there is no evidence of volcanism or sea areas as on Mercury. There is evidence of Mercury still slowly compacting and it exhibits settling quakes. Mercury exhibits a very weak magnetic field of $\approx 1 / 100$ that of the Earth which may be a frozen in remnant of a time in which Mercury could have initially exhibited a dynamo to cause a short term active magnetic field. The relatively large metallic conducting core of Mercury would then be subject to magnetic braking due to the magnetic field of the Sun. From its proximity to the Sun the solar wind would be expected to have stripped away any initial atmosphere and water as the initial magnetic field dissipated.

It is argued that the lower average density of Venus precluded the formation of any liquid conducting core region so that Venus then never manifested any magnetic field structure. The Earth because of the anomalously high average density structure did manifest a liquid rotating conducting core region and hence its own magnetic field. Farther out in the solar system Mars developed an incompletely differentiated structure with a relatively poor electrical conductivity in the core due to the rapid cooling of the smaller planet. The greatest consequence of Earth developing a magnetic field structure is that a long-term mechanism for moving heat from the interior to the surface known presently as plate technics was established. The importance of the Earth-Moon formation process is that the process allowed a magnetic field and the formation of plate tectonics to be developed on Earth as an exceptional event. An implication is that for planets to form and be capable of plate tectonics is an exceptional event. So that the vast majority of planets can be expected to form without any long-term gradual release of interior heat. The development of complex life forms through evolution takes long term sequential development stages. Absent any long-term heat release mechanism life expectation due to long term evolution is doomed. The entire surface of Venus shows random cratering indicating that the heat release mechanism was entire surface convulsions [7]. This is the heat release mechanism that happens in the absence of plate tectonics.

One of the purposes of this paper is to illuminate how the presence of the Sun's magnetic field influenced the rotation of the terrestrial planets in our solar system. The development of the planets Mercury, Venus, Earth, and Mars suggest tests that can be used to validate the model. One of these tests involves the interaction of the planet's rotation within the Sun's magnetic field. The Sun's magnetic field manifests both an expected dipolar magnetic field as well as open magnetic field loops that extend far out away from the Sun. The Sun is currently $4.6 \pm 0.1 \mathrm{BYr}$ old. The Sun's luminosity has gradually been increasing as shown in the background information chart, but most properties of the Sun have been 
remarkedly stable over its lifetime. The amazing fact is that this massive and ancient star currently exhibits a dipolar magnetic field component that reverses in an approximately 11-year cycle. Assuming for the moment that the rate of flux reversals started early in the Sun's formation, that gives an estimated $4 \times 10^{8}$ flux reversals over the Sun's lifetime. The location of Sun's poles is where the magnetic field lines are the most concentrated and where the magnetic braking effects should be the strongest. Consequently, the equatorial regions of the Sun are expected to rotate faster than the polar regions where the field is more concentrated, as is observed. The rotation of the Sun causes an outer spiral pattern in the nearly radial component of the Sun's magnetic field in the plane of the rotation known as the Parker Spiral [8], as named after Eugene Parker. This is depicted in Figure 12. This spiral pattern causes a drag that is usually credited with transferring angular momentum away from the Sun. Presently a probe called the Parker Probe is starting to study the magnetic field changes in closer proximity to the Sun. This drag is expected to be most active in the early proto-planet solar system when the gas and dust that later formed the planets constituted a conductive plasma. Later as the planets formed the flux reversals in the dipolar magnetic field can then interact with the electrical conducting core regions of the terrestrial planets. This interaction depends on the relative conductivity of the planet core and the presence of planet magnetic fields that add as vectors with the Sun's magnetic field. Just as a rotating conducting aluminum disk experiences a magnetic braking effect that rapidly slows the rotation rate, the planet cores also experienced a braking effect dependent on core conductivity and the presence of local magnetic fields. Reversals in the Sun's dipolar field cause time varying flux reversals that act to slow the core rotations in the absence of planet magnetic fields. The flux is proportional to the strength of the Sun's magnetic field at the planet location multiplied by the core cross section area and divided by the flux reversal time. Presently the flux reversal time is about $11 \mathrm{yr}=3.5 \times$ $10^{8} \mathrm{sec}$. This time is very short compared to any planet development time so that a great many flux reversals and slowing impulses act on electrically conducting

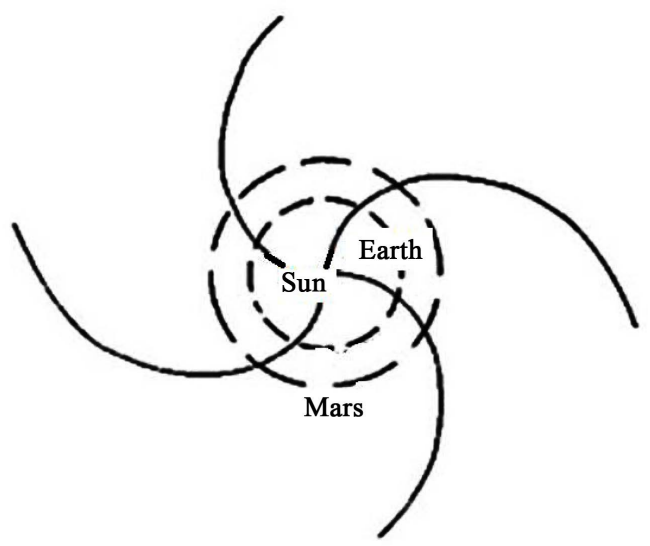

Figure 12. Parker magnetic field spiral depicts the path of the open magnetic loops due to the sun rotation. Region out to about mars is depicted. Figure from Ref. [8]. 
core regions. Thus Mercury, once any initial magnetic field was frozen out, was limited to only a slow rotation. Venus which never acquired its own magnetic field was also limited to only a slow rotation due to the Sun's changing dipolar magnetic field. Earth only developed a magnetic field of its own because of the pre-Earth pre-Moon interaction shown in Figure 10. Mars's small size limited the planet differentiation so that the core electrical conductivity was limited. Thus Mars's rotation was not limited by the changing magnetic flux from the Sun.

Thus the rotation rate of Mercury and Venus are both expected to be quite slow. The rotation rate of Mercury is slow with the orbital period to rotation period exhibiting a 3:2 orbital to rotation ratio (Table 1). The eccentricity of Mercury is the largest of all the planets at a value of 0.21 so the distance of closest approach rotates about the Sun (Table 1). The spin axis is very nearly perpendicular to the plane of the orbit about the Sun (Table 1). Mercury is the only planet that requires general relativity corrections to precisely calculate the orbital precession. Venus with a large conduction core itself, but absent its own magnetic field (Table 1) must exhibit only a slow rotation due to its interaction with the Sun's magnetic field. As the atmosphere thickened from some initial state, atmospheric drag has resulted in a slightly reverse rotation from that of the other terrestrial planets. The rotation axis of Venus points only about $3^{\circ}$ away from a perpendicular to the orbital plane so that little seasonal effects are expected (Table 1). Earth has its own magnetosphere which interacts with the Sun's field largely shielding the Earth from the effects of the Sun's dipole magnetic field, as well as from the solar wind emanating from the Sun. The initial rotation rate of the Earth resulted from the obliqueness of the collision of the pre-Earth and the pre-Moon object. The initial Earth rotation rate then changed to preserve the Earth-Moon angular momentum as depicted in Figures 6-8. The eccentricity of the Earth orbit about the Sun exhibits only a small eccentricity of 0.02 (Table 1). The Earth's rotation axis is tilted at $23.5^{\circ}$ away from the plane of the Earth's orbit about the Sun so that seasonal effects are expected. There is also a precession of the Earth's rotation axis with a period of about 26,000 years so that certain long-term recurring climate effects are expected.

Mars exhibits a reddish color due to iron oxide being scattered about the surface indicating incomplete gravitation differentiation. This implies that the core exhibits a relatively poor electrical conductivity that limits the core interaction with the Sun's magnetic field since Mars does not exhibit its own magnetic field. The rotation rate of Mars is not then strongly slowed by the Sun's magnetic field so that the rotation rate of Mars has persisted largely unchanged over billions of years. The rotation rate of Mars at the present time closely matches that of the Earth, but as shown in Figures 6-8 the rotation rate of the Earth in early times was faster than that of Mars, and in the future, the rotation rate of the Earth will be slower than that of Mars. Currently, Mars has an inclination angle of $\approx 25^{\circ}$ so that Mars is expected to exhibit seasonal changes similar to the Earth (Table 1). But since Mars does not have a large moon to maintain a particular rotation axis 
through the conservation of angular momentum, there is evidence that the location of the poles and ice cap regions have shifted over long periods of time. Since large concentrations of ice are expected in the vicinity of the poles, large concentrations of water may be expected to be found at different locations below the surface of Mars.

\section{Conclusion}

The Earth-Moon formation event implies that this formation event was required for Earth to exhibit a magnetic field early on, and that Venus and Mars did not exhibit initially a global magnetic field. This is different from the usual analogy to Earth that these planets initially exhibited magnetic fields and then subsequently lost those magnetic fields. The different rotation rates of the terrestrial planets correlate with the expected interaction of the planet cores with the time varying dipole field of the Sun. Only Earth with a magnetic field would exhibit a mechanism for long term heat release through plate tectonics. Vulcanism and the attendant plate tectonics are now becoming widely recognized as being necessary for the long term evolution of complex life forms [9]. The end result is then that the very early formation period of the Earth-Moon system was necessary for the subsequent development of complex life forms on planet Earth. In searching for life on exoplanets, it is then best to look for terrestrial worlds with a sizable moon; a very small subset of those observed.

\section{Acknowledgements}

Helpful discussions with George R. Hendrey and Steven A. Schwarz are gratefully acknowledged.

\section{Conflicts of Interest}

The author declares no conflicts of interest regarding the publication of this paper.

\section{References}

[1] Giant-Impact Hypothesis, Wikipedia. This Article Summarizes Various Formation Processes for the Earth as Resulting from a Collision between a Pre-Earth and Another Impactor. 62 References Are Indicated. The Differences Have Little Impact Here Since This Article Deals with the Resultant Earth and Moon as a Coupled System.

[2] Carroll, B.W. and Ostlie, D.A. (2007) An Introduction to Modern Astrophysics. ${ }^{\text {nd }}$ Edition, Pearson, San Francisco, p. 350.

[3] Application of Eq. 19.4 Yields a Roche Limit of 2.9 R Earth for the Earth-Moon System (Ref. 2, p. 724).

[4] Johnson, B.W. and Wing, B.A. (2020) Limited Archaean Continental Emergence Reflected in an Early Archaean ${ }^{18} \mathrm{O}$-Enriched Ocean. Nature Geoscience, 13, 243-248. https://doi.org/10.1038/s41561-020-0538-9

[5] Only a Subset of the NASA Entries Are Listed. 
https://solarsystem.nasa.gov/planet-compare/

[6] Hazen, R.M. (2012) The Story of Earth. Penguin Books, Chapter 7.

[7] https://nineplanets.org/venus/.

[8] Parker, E.N. (1958) Dynamics of the Interplanetary Gas and Magnetic Fields. Astrophysical Journal, 128, 664. https://doi.org/10.1086/146579

[9] Rebecca, B. (2018) Plate Tectonics May Be Essential for Life. Quanta Magazine. Lee, P. (2009) Plate Tectonics Could Be Essential For Life. AstroBiology Magazine. Shannon Hall, Space (2017) Earth's Tectonic Activity May Be Crucial for Life and Rare in Our Galaxy. Scientific American. 


\section{Appendix}

This appendix illustrates that the gravitational potential energy of the FedEx arrow shown in the text is greater when it orbits the Earth pointed up away from the Earth than when pointed down toward the Earth. This will be illustrated with specific numbers for ease of illustration. The reasonable assumption will be made that the dimensions of the composite FedEx arrow are small compared to the distance, $D$, from the center of mass of the Earth to the center of mass of the FedEx arrow. In the diagram, the location of the peak of the equilateral triangle has the coordinates $(0,746.4 \mathrm{~m})$. The location of the center of mass of the lower rectangle has the coordinates $(0,200)$ and the center of mass of the upper equilateral triangle has the coordinates $(0,515.5)$ with distances measured in meters for convenience. The thickness of the FedEx arrow is assumed to be 1 meter with a density of $1 \mathrm{~kg} / \mathrm{m}^{3}$. The mass of the rectangle is then $8.00 \times 10^{4} \mathrm{~kg}$ and the mass of the triangle is then $6.93 \times 10^{4} \mathrm{~kg}$. The center of mass of the composite FedEx arrow is then at $(0,346.4 \mathrm{~m})$ in the diagram. The distance from the center of mass of the composite object to the center of mass of the triangle is then 169.1 $\mathrm{m}$. The distance from the center of mass to the center of mass of the rectangle is then $146.4 \mathrm{~m}$.

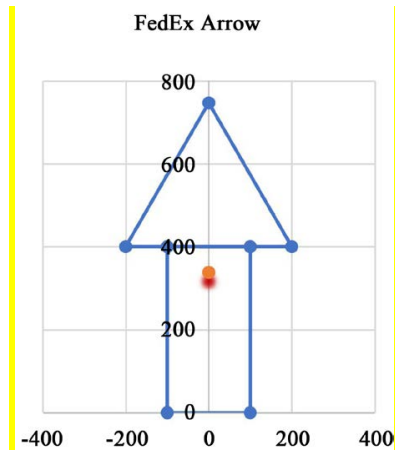

The gravitational potential energy when the FedEx arrow points up away from the Earth can then be defined:

$$
U_{u p}=-G \cdot M_{E}\left[\frac{m_{t r i}}{D+169.1}+\frac{m_{\text {rect }}}{D-146.4}\right]
$$

where $G=$ the universal gravitational constant and $M_{E}$ is the mass of the Earth, $D$ is the distance from the center of mass of the Earth to the center of mass of the composite object, $m_{t r i}$ is the mass of the triangle, and $m_{\text {rect }}$ is the mass of the rectangle. The distances are in meters and $D$ is much greater than the dimensions of the FedEx arrow. Similarly, the gravitational potential energy with the FedEx arrow pointing down toward the Earth can be written as:

$$
U_{d n}=-G \cdot M_{E}\left[\frac{m_{t r i}}{D-169.1}+\frac{m_{r e c t}}{D+146.4}\right]
$$

It is then desired to show that $U_{u p}$ exceeds $U_{d n}$, or equivalently that $U_{u p}-U_{d n}>$ 0 . To do this it is useful to make use of the approximation that $D \gg$ the di- 
mensions of the FedEx arrow. $U_{u p}$ can then be written as

$$
\begin{aligned}
U_{u p} \approx & -\frac{G \cdot M_{E}}{D}\left[m_{\text {tri }} \cdot\left(1-\frac{169.1}{D}+\left(\frac{169.1}{D}\right)^{2}-\left(\frac{169.1}{D}\right)^{3}\right)\right. \\
& \left.+m_{\text {rect }} \cdot\left(1+\frac{146.4}{D}+\left(\frac{146.4}{D}\right)^{2}+\left(\frac{146.4}{D}\right)^{3}\right)\right]
\end{aligned}
$$

And $U_{d n}$ can be written as

$$
\begin{aligned}
U_{d n} \approx & -\frac{G \cdot M_{E}}{D}\left[m_{t r i} \cdot\left(1+\frac{169.1}{D}+\left(\frac{169.1}{D}\right)^{2}+\left(\frac{169.1}{D}\right)^{3}\right)\right. \\
& \left.+m_{\text {rect }} \cdot\left(1-\frac{146.4}{D}+\left(\frac{146.4}{D}\right)^{2}-\left(\frac{146.4}{D}\right)^{3}\right)\right]
\end{aligned}
$$

The difference then becomes

$$
\begin{gathered}
U_{u p}-U_{d n}=\frac{G \cdot M_{E}}{D^{1}} \cdot\left[2 \cdot \frac{169.1}{D} \cdot m_{t r i}-2 \cdot \frac{146.4}{D} \cdot m_{r e c t}\right. \\
\left.+2 \cdot\left(\frac{169.1}{D}\right)^{3} \cdot m_{t r i}-2 \cdot\left(\frac{146.4}{D}\right)^{3} \cdot m_{r e c t}\right] \\
U_{u p}-U_{d n}=\frac{G \cdot M_{E}}{D^{1}} \cdot\left[2 \cdot \frac{169.1}{D} \cdot m_{t r i}-2 \cdot \frac{146.4}{D} \cdot m_{r e c t}\right. \\
\left.+2 \cdot\left(\frac{169.1}{D}\right)^{3} \cdot m_{t r i}-2 \cdot\left(\frac{146.4}{D}\right)^{3} \cdot m_{r e c t}\right] \\
U_{u p}-U_{d n}=\frac{G \cdot M_{E}}{D^{1}} \cdot\left[2 \cdot\left(\frac{169.1}{D}\right)^{3} \cdot m_{t r i}-2 \cdot\left(\frac{146.4}{D}\right)^{3} \cdot m_{r e c t}\right] \mathrm{m}^{3} \\
U_{u p}-U_{d n}=\frac{G \cdot M_{E} \cdot\left[2 \cdot(169.1)^{3} \cdot m_{t r i}-2 \cdot(146.4)^{3} \cdot m_{r e c t}\right] \mathrm{m}^{3}}{D^{4}} \\
U_{u p}-U_{d n}=\frac{G \cdot M_{E}}{D^{4}} \cdot\left[6.70 \times 10^{11}-5.02 \times 10^{11}\right] \mathrm{kg} \cdot \mathrm{m}^{3}
\end{gathered}
$$

which is $>0$.

Notice that what maybe considered the linear term, as well as the squared term, dropped out. It should be noted that the linear term was used to find the center of mass of the object.

Thus it has been shown that the lowest gravitational potential energy corresponds to the FedEx arrow orbiting the Earth with the arrow pointing down toward the Earth. 